\title{
Introduction
}

\section{Models in Spatial Analysis}

\section{Which model and which spatial analysis?}

The spatial dimension plays a key role in many social phenomena. On the one hand things are unequally distributed through space, creating spatial differentiation, segregation, discontinuities. On the other hand there is a feedback loop between a society's organization and a space's configuration. Most of the time, the modeling of spatial phenomena and processes requires the combination of knowledge and skills from various fields, some involving the theme of research, others originating from computer science, statistics, physics or mathematics. These latter subjects are relevant because they provide stimulating methodological prospects to theorists interested in the organization of space and the evolution of its structures (whether they be geographers, urban planners, archaeologists, ecologists, agriculture scientists, etc.). Besides, they also offer useful technical frameworks for formalizing the thematician's theoretical models. On the other hand, the latter provide problems and data that can be used to implement and test models designed by mathematicians, statisticians and computer scientists. In such context the phrase spatial model takes on different meanings. Even though researchers in different fields may agree on a relatively broad definition of the concept of a "model" as "a schematic representation of reality, developed with the goal of understanding and explaining it" ${ }^{\prime 1}$, the referents are significantly different from one subject to another.

In order to illustrate the diversity of meanings that come with the word "model" in a given application, in the following sections we will rely for the most part on two concrete examples. In the first, the goal is to modelize the evolution of the spatial distribution of the population over a few decades, and in the second, of the land use . From a methodological perspective, each of these two examples involves dealing with and modeling "spatial dynamics". However, modeling spatial dynamics can

1 A definition close to that of P. Haggett [HAG 65] used by F. Durand-Dastès in Chapter 1. Introduction by Lena SANDERS to "Models in Spatial Analysis", ISTE, London, 319p. 
take on several different meanings: it can consist of describing changes as clearly as possible, or of finding causality underlying the type and speed of the observed evolution. These two approaches, the former used to describe and the latter to explain, should logically complement each other and be conducted one after the other. In practice, however, the line between the two is not always clear, and the structure of the information itself leads to different attitudes in research. If this structure is complex, the first step can constitute the entire work of modeling. Indeed, the objective is then to provide a simplified picture, in the form of a map, for example, of a complex information obtained from several sources. If, on the other hand, the information is described by a simple and relatively regular structure, such as in the case of statistical information, the first step is short and most of the modeling work is done in the second. In order to study spatial differentials in population growth, the geographer will build an explanatory model based on mechanisms he knows are fundamental, the role of rent, the effects of competition for space, urban polarization, the deterring effect of distance, etc. In order to study the evolution of land use, the agriculture scientist will develop a model involving the modes and structures of farming, the specializations and quality of soil.

In any case, the first step consists of identifying the elementary objects that give the level at which the information has to be collected. These objects can be cells or pixels (image analysis), households or firms (surveys or census results), or spatial entities (morphological or administrative units). When studying a settlement system, the elementary entities can be municipalities or agglomerations. When analyzing the land use, plots or farms can be used. This identification becomes more complicated in a dynamic framework, since there can be changes in the object's semantics (when a given agglomeration "absorbs" another, for example), identifier (a plot can be divided in two) or its spatial extent (expansion of a farm) [CHE 95]. Once the objects are identified, we have to come up with ways of measuring changes that make sense in regards to the topic, and which are compatible with the spatial scale. Already, throughout this entire process of formalizing information, we are dealing with models. These are, on the one hand, conceptual models concerning the meaning of the chosen indicators, particularly the link between the phenomenon being studied (which refers to a theoretical framework) and the set of data obtained from its measurements (which refers to what is observable), given the observation levels that were chosen. These are, on the other hand, models of data that are necessary to organize thematic and geometric information in order to process them (relational, object-oriented, vector/raster etc.).

Once the thematic model has been designed and the gathered information has been organized, it is necessary to formalize the model in order to validate it, to test hypotheses and, sometimes, to establish scenarios to conduct prospective studies or to help decision making. Different approaches are then possible, each one associated with a specific set of skills. 
If we choose a geomatics approach, we would develop and use the functionalities of a geographic information system (GIS) in order to connect layers of information related to different aspects of the phenomenon being studied and to find evidence of possible spatial regularities. We can construct, for example, requests designed to pinpoint plots located at a certain distance from a freeway interchange and that have undergone a specific change in land use. If we choose a statistical framework, a typical method consists of identifying the variables that have to "be explained" (dependent variables), quantitative ones (population growth rate, variations in the number of jobs) or qualitative (changes in land use, changes in the economic profile), and "explanatory" variables that refer to the features of the agglomerations or land plots and express the hypotheses set out in the thematic model. For the statistician the discussion will focus on the choice of a statistical model, linear, loglinear, exponential, etc., depending on the statistical nature of the variables and on their relations. Facing the same question, researchers trained in mathematics or physics will develop a dynamic model in the framework of complex systems (for example, a non-linear prey-predator model) that includes bifurcation properties. These will be particularly useful if we consider that the parameters that characterize the general environment of the system being studied are governed by threshold effects that may cause a qualitative change in the behavior of settlement dynamics. Formalizing the model within the framework of computer sciences will lead to the use of cellular automata or multi-agent systems and to focus on interaction and emergence properties. Such models work by conducting simulations, and these are particularly useful when trying to establish the effect that the set of local interactions has on the evolution of spatial structures at a higher level ([WHI 97], [BEN 04], [POR 04]).

To the thematician, these different approaches represent a series of methods that are useful to formalize and test his thematic model, each one leading to different and complementary possibilities. His choice will depend on his abilities, his collaborations or, better, on the adequacy between his hypotheses on the why and the how of the change he is interested in and the properties of the available statistical, physical, geomatics or computer model. In some cases, he may have to use a series of models: a GIS to create spatial indicators; a statistical model to test the relevance of the relationship between some of the variables used, or also to estimate missing information; a differential equation model to deal with the overall evolutions of a certain number of state variables; a cellular automata model to simulate the local spatial interactions; a multi-agent system to account for the effects of a cooperation between several agents. In order to implement a thematic model, it thus becomes necessary to string together, to couple and to integrate models originating from different fields, and confusions of course can occur when the same word "model" is used without being associated with a complement that specifies its meaning. 
Each of these groups of methods has its specific advantages and integrates in its own way the spatial dimension of the phenomena being studied. The statistical approach emphasizes the covariation mechanisms of the phenomena, and space can be included in the analysis by means, first, of the type of statistical individuals considered (spatial entities, location pairs) and, second, of the variables (distances, shapes of spacings or types of neighborhoods, for example). In dynamic models, space can, in the same way, intervene as a frame for the studied objects (spatial entities), and also through an interaction term, connecting the dynamics of the entities with each other (the distance or the commuting cost between two entities, for example). If the cellular automata are used to represent geographic space, the very driving force of the change is spatial, since any change can be determined based on the neighborhood configuration. Each of these methodological frameworks can be used with the goal of describing, exploring or explaining. The lines that separate these approaches are not always clear and depend more on how the researcher designs his methodological device than on the methods themselves.

The question can be stated in similar terms for spatial analysis. Depending on the contexts and the authors, different meanings are bound to this concept in the case of geography alone. Some give the phrase a strong thematic meaning: "Formalized analysis of the configuration and properties of the geographic space, as it is produced and experienced by human societies" [PUM 97]. Others emphasize the spatial aspect of the phenomenon being studied: "Searching, within the features of spatial entities, what is relevant to their geographic positions, particularly their relative geographic positions, making it necessary to model the spatial structure" [CHA 95]. Others, finally, insist on the methodological aspects, and spatial analysis refers to the "set of mathematical and statistical methods designed to specify the nature, the quality, the quantity related to places and to their relations - all of this defining space - by studying both attributes and localizations" [BRU 92], or also, in a similar approach, a "whole cluster of techniques and models which apply formal, usually quantitative, structures to systems in which the prime variables of interest vary significantly across space" [LON 96]. GIS users, on the other hand, when they use the phrase spatial analysis, refer to a set of functionalities, such as superposition techniques, topological and geometric functions, interpolation techniques, network analysis, etc.

Again, there can be some confusion between thematicians, such as geographers, who see spatial analysis as a conceptual framework in which the effects of position, neighborhood and co-occurrence play a key role in helping to make clear the interactions between space and society, associated with elementary spatial models referring to gravity, potential, diffusion and to the tools of spatial statistics (for example, spatial autocorrelation, variogram), and GIS users for whom the phrase spatial analysis sometimes means the same thing as the geometric and superposition functionalities of GISs. 
Thus, a computer scientist and a thematician do not share the same meaning for the phrase "models in spatial analysis". We will not discuss in detail in this book the differences in points of view, but instead we will adopt the following statement. The idea is to describe the approaches concerned with thematic modeling, and to give an idea of the diversity of the methodological choices made in practice by those whose object of study is strongly rooted in space. Any modeling requires geographic information to be formalized, but the emphasis does not rest explicitly on this question, but rather on how the hypotheses are formalized. The goal is to understand, explain and simulate spatial organizations, special localizations, the emergence or the end of certain phenomena in certain locations, and the types of interactions between different places.

Geographic information plays a central role in all of the studies detailed in this book, even if there are few explicit references to GISs. Despite going unnoticed in these modeling studies, they have nonetheless a strong implicit presence and are essential tools in some processing steps. Certain spatial analysis methods useful for developing a thematic spatial model exist in the GIS, or can easily be internalized, while others are used in completely independent ways or are coupled. Geographers have produced remarkable advances this last decade in coupling and integrating tools of sptial analysis, simulation models and GIS ([MAG 06]).

Each chapter in this book shows the relationship between a conceptual framework of spatial analysis and a set of methods and models. The frameworks are related to the questions of localizations and interactions in space, of geographic scales and levels of observation, of spatial dynamics. Some authors favor the contribution of a group of methods originating from statistics, mathematics, and computer science, while others base their presentations on theories deeply rooted in geography or economics. All of them attach a key importance to the connection between the thematic meaning of their studies and the properties of the tools they have chosen. Space is present both as an object - the objective being to describe, understand and predict localizations, spatial configurations and their evolution - and as a part of the explanation - the objective being to assess the effects of location and neighborhood on these localizations, configurations and changes.

\section{Two central and recurring concepts: spatial interactions, levels of observation}

Every chapter in this book deals with situations where space plays a key role, sometimes as a passive frame but most often as an active function. On the other hand, the various chapters are very diverse in terms of the phenomena being studied (for example, water runoff, localization of public services, population mobility), of the analysis tools (for example, multivariate analysis, differential equations, multiagent systems), of spatial scales (from a square foot cell to the national level) and 
time scales (from a minute to a century). This book thus illustrates the diversity of questions and practices in spatial analysis modeling. Beyond this diversity, there are recurring concepts that are unavoidable in any approach in terms of spatial analysis. These concepts are spatial interactions and levels.

\section{Modeling spatial interactions}

The concept of spatial interactions is used in most chapters, and is in fact at the core of the spatial analysis approach. When the locations have been characterized, when the intensity and the types of spatial differentiations have been determined, and when the similarities and contrasts have been brought to light, the next task consists of finding the relationship between these features of spatial organization and the exchanges these locations maintain among each other, as well as the mutual influences they have on each other. These influences and exchanges originate from the disparities of space; in fact A. Franc uses the phrase "interactions driven by spatial organization" (Chapter 9). The other way round, these interactions, in turn, contribute to producing disparities. Thus, spatial interactions reflect relations of complementarity and/or competition between the locations and act as a driving force in the transformation and the dynamics of spatial systems. The interaction between these locations can either be the object of research itself (when trying to explain why some flows are more or less significant between certain pairs of locations), or represent a constraint (movement from one location to another is viewed as a constraint on achieving a task for a given agent), or also constitute the explanatory element of a dynamic (the start of or an increase in a phenomenon in a location brought about by exchanges with other locations).

As a result, spatial interactions take on various forms, operate on several levels, and their analyses lead to different formalizations. In the following sections, we will attempt to express the major aspects of this diversity that are dealt with throughout the different chapters.

\section{Exchanges between pairs of locations}

In a first set of models, locations have symmetrical roles, because each location has the possibility of being a transmitter and a receiver. When studying interregional migratory exchanges, for example, each considered area represents a possible settling location for migrants from elsewhere and, at the same time, is the departure location for a certain number of inhabitants who have decided to migrate. The most common spatial interaction models are designed to express the intensity of the flows or exchanges between an original location and a destination, depending on the characteristics of these locations (in terms of departure potential and attractiveness of the destination) and on the deterrent effect of the distance between the two 
locations. F. Durand-Dastès (Chapter 1) uses the gravity model, one of the most common in spatial analysis, to account for what he refers to as the "universals" of modeling. In more general terms, the author stresses the connection between interaction and causality, and underlines the importance of considering spatial interaction for the choice of a type of systemic formalization in spatial analysis.

Exchanges between locations can be material or immaterial, they can be expressed in terms of their quantity (number of migrants) or their nature (presence of a connection by air), and they can be described using sagittal, chorematic or matrix models. The combination of all the spatial interactions operating on an elementary level sometimes leads to the emergence of complex spatial structures. P. Frankhauser and D. Pumain analyze the contribution of fractals to the study of such structures (Chapter 10).

\section{Exchanges between locations with different statuses}

A certain number of interactions, on the other hand, are dissymmetric from a semantic perspective, and explicitly involve differences in status between the locations that are considered. Such is the case for diffusion phenomena, in which one or several places are considered as favored transmitters (of information, an innovation, a disease) whereas the other places constitute locations for the receivers, the adopters, the contaminated. This is also the case when considering consumers, characterized by where they live, searching for a localized service: school, day care center, hospital, etc. Depending on the problem, different formalizations are possible. The first thing to do is to determine on what geographic level the starting place (the location with access to the innovation or accomodating the population expressing a potential demand) should be considered: building, block, city or a larger area (in practice, an administrative district most of the time). Destination locations can be identified as entities of the same nature, or simply defined by localized points in the case of a service for example. We then have to determine an adequate model to describe the associated interactions and their consequences on the organization of space and on its dynamics.

In Chapter 3, D. Peters and I. Thomas present models that can be used to determine the optimal location of public services given the distribution of the population concerned by these services. Rather than using the word interaction, they suggest the concept of spatial adequacy between the supply and the demand for these services. Chapter 5, by Th. Saint-Julien, focuses on the spatial diffusion phenomena and on how they are formalized, the idea being that a certain number of transformations of space are due to contagion effects. The phenomenon may be physical, such as the propagation of a plant or a disease, or social, by means of imitation behavior, in areas as diverse as innovation in agriculture or architecture, and behaviors in terms of fertility. The most common formalizations, developed in 
the 1950 s, remain valid today on a conceptual level, with much broader possibilities for technical applications.

In Chapter 4, S. Chardonnel shows how the principles of time-geography are used in order to simulate the impact of a change in public transportation on the individual possibilities of achieving space-time trajectories. In this case, spatial interaction is in the form of commutes, for example "home - day care center workplace - day care center - home", in accordance with certain constraints on the individuals' schedules and how long they have to stay in certain locations. The point of view is that of the individual subject to constraints in space (reaching a destination while stopping at certain required stops, for example, the day care center) and in time (observing the opening hours of the day care center and work hours).

Chapters 3 and 5 illustrate how, faced with the similar problem of access to a service, the model depends on the research prospects: in one case, the objective is to optimize the localization of a set of recycling centers, while in the other, the access to day care acts as a constraint on the home-to-work commutes of individuals. In the first case, an optimization model is used to provide the distribution of recycling centers that leads to minimize the sum of individual trips. In the second, the locations of the home and day care centers are set, and simulations based on logical rules, involving schedules and lengths of time, are used in order to test the impact of improvements on public transportation, in terms of frequency, on the range of individual possibilities.

\section{Influence of a location on neighboring locations}

Spatial interactions can also be expressed as an influence of a location on another, without being explicitly embodied in the form of a measurable exchange or flow. Cellular automata are often used to formalize the effect of such influences on local change and simulate the spatial configurations that arise at a global level. In Chapter 8, F. Dureau and C. Weber present a model for land use change at a pixel level. The land use is identified based on the analysis of a satellite picture, then the potential for change in land use is determined for each pixel based on land use in the neighboring pixels, the intensity of the influence decreasing with distance. The authors used this model to simulate the expansion of construction on the outskirts of Bogota.

H. Mathian and M. Piron (Chapter 2) have also focused on the formalization of the consequences of proximity relations. These relations are the result of similarities or, on the contrary, of oppositions between the locations, and the associated spatial structuralization can be determined from local statistics. Such influences can work according to a logic of location pairs (what happens in location A depends on the 
characteristics of location B) or according to a logic of context (what happens in location A depends on the general characteristics of the environment where $\mathrm{A}$ is located). The authors show how the statistics formalism contributes to describing and sometimes explaining the result of this influence system on the intensities of the spatial differentiations. A mathematical formalization of the same phenomena is given in Chapter 9, focusing more on the dynamic aspects of the interactions. The geographic coordinates of the spatial entities described by the model then play the role of parameters in the space-time system under study.

\section{Interaction between agents, groups of agents and the environment}

Throughout the previous section, we have focused on spatial interactions as interactions between locations. This phrase, which relies mostly on an aggregate logic, is used in order to emphasize spatial logics, but clearly these interactions between the locations take shape most of the time as exchanges between agents who live and act in these locations: these are exchanges between individuals, or between individuals and groups, or also between individuals and firms or institutions. Chapters 4, 5 and 6 give great importance to individuals and deal with interactions on this level more explicitly. Thus, E. Holm and L. Sanders examine the interactions that drive the decision to migrate and which should be integrated into a microsimulation model: interactions of the individual with other members of the household, interactions with the agents comprising his environment (building, neighborhood, city), and finally, the relationship with the locations where he stopped along his own past trajectory (Chapter 6).

Another form of interaction is modeled by several authors: that which exists between individuals or groups and the environment. Most of the time, these interactions occur in a given space, but they are generally not described in terms of spatial interactions. These man-environment or society-nature interactions are discussed more explicitly in Chapter 7, where J.-P. Treuil, C. Mullon, E. Perrier and M. Piron explain the use of multi-agent systems to simulate spatialized dynamics, the driving force of change operating mainly through interactions between agents. The entire functional diversity of interactions is considered: solving computer science problems, simulating negotiation and cooperation problems between agents, managing the evolution of artificial worlds comprised of agents and resources that interact.

From the individual to the group, from the pixel to the area, from the element to the aggregate: the question of the level of observation and modeling

There are two major questions in this field: what is the adequate level of observation given the question we wish to answer? How do we go from one level to 
another, in terms of semantics and methodology? Of course, no chapter can avoid facing the first question, and several of them explicitly deal with the second.

\section{Choosing the level of observation}

The first step in many research studies consists of determining the best level of observation to choose, given the objective of the study. How the question is stated can sometimes lead to an obvious answer: if we wish to know what determines individuals in their choice of transportation from their home to their workplace, we have to work on the individual level; the same goes when focusing on the behavior of working parents and how they manage their daily space-time (see Chapter 4); on the other hand, if we study how an administrative or linguistic border can act as a barrier for migrating patterns, an aggregate level is preferable. Other questions lead to a large variety of possible choices. Such is the case when trying, in an operational process, to determine the localization of a public service (see Chapter 3). The question is to understand, given the distribution shape of the population expressing a need, as well as equity and cost criteria, what spatial aggregate level to choose in order to reach optimal localization. For other questions, finally, there can even be some controversy among researchers as to what level of observation and modeling to choose. In different subjects, there is also a debate between the advocates of modeling on the most basic level and those who suggest developing an aggregate model to describe the dynamics of spatial aggregates (see Chapters 6 and 7). In order to understand the dynamics of water run-off, should we track the "drops of water", or is it enough to formalize the question in terms of flows? In order to describe the dynamics of population in a set of municipalities, should we formalize the migration choices of individuals or attempt to model the intermunicipality regularities in terms of residential appeal? The problem commonly amounts to choosing between the individual level and the spatial aggregate level, but it also arises, sometimes in a more subtle form, when choosing a level of territorial organization over another (see Chapter 2).

\section{Articulating different levels of observation and modeling}

In a certain number of cases, the question is not what choice to make, but instead how to simultaneously use several levels in a heuristic perspective. Thus, the very meaning of both the attributes and the questions asked can vary when covering the different levels of the geographic scale. The authors of Chapter 2 give an overview of the different ways to approach several levels of observation in the context of a study. One method can be to compare the distribution of a phenomenon with respect to different segmentations of space, since such a comparison can help determine the scales of the spatial differentiation being studied (of different forms of segregation for example). It is also possible to compare the type of a statistical relation between two phenomena when changing the level of observation. This relation can be stable or instead reveal stark changes. This last case is problematic from a statistical point 
of view, since the results depend on which level of observation is chosen [FOT 91], but it provides information on how the spatial differentiations are structured, on the scales of operation of the different combinations of factors being studied.

A similar question is discussed in Chapter 9 with the concepts of scale transfer and "perfect aggregation". The author mentions a common practice in research, in plant ecology for example, consisting of developing a model based on data that are valid on fine scale in order to answer questions on a much broader level. $\mathrm{He}$ underlines the gap between the level on which a model is valid and the level for which data is available. This type of approach requires specific knowledge of the relationships between scales, both from empirical and theoretical perspectives. The perfect aggregate from a mathematical perspective (implying for example that if the calculated averages on two sets of elements are equal at a given time, they will remain that way) is rarely observed in practice. When modeling the dynamics of a spatial model, the opposite is often assumed, meaning that spatial differentiations act as a driving force in the evolution of a system. The focus is on the emergence of new spatial differentiations and the change from one scale to the other can then no longer be formalized in a simple way. Computer modeling, particularly the use of multiagent systems, offers a greater flexibility for managing interactions between objects from different levels, a flexibility that comes at the price, however, of greater difficulties for validation (see Chapter 7).

The problem of interlocking scales is also at the core of the fractal approach. This method focuses on the mechanisms that lead to similar structures on different levels of territorial organization. The authors of Chapter 10 present several methods designed to characterize such structures from a geometric point of view and, most importantly, to locate thresholds in space that correspond to the change from one type of structure to another (a useful method for determining the lines separating different types of peri-urban built up areas, for example). The fractal approach also usually makes it possible to find the connection between the behavior of a non-linear dynamic system and the type of objects produced by this system. The different steps presented in this chapter are therefore also very useful for characterizing and comparing the structures simulated using dynamic models with the spatial structures that are observed.

Another approach consists of simultaneously formalizing, within a same process, mechanisms related to different scales. They can be integrated into one same model, with for example migration choices formalized on the level of individuals, and residential appeal formalized on the level of municipalities (see Chapter 6). It is also possible to pair up models operating in parallel, for example, by simulating on one hand the potential for change in land occupation in one cell based on the neighborhood, and on the other hand the preferences of individuals for residential 
choice (see Chapter 8). In each case, the difficulty lies in having to make hypotheses on the type of the feedback between the two levels that are considered.

Multi-scale approaches and the modeling of spatial interactions thus constitute the major themes of this book. Although they deal with different topics and objects, the authors all deal with questions about the organization of space and its role in the dynamics of the phenomena that are studied. The diversity of backgrounds among the authors and of the ways of approaching the problem of space gives an idea of how broad the field of spatial analysis is, and shows the advantage in this field of an interdisciplinary perspective.

\section{Bibliography}

[BEN 04] Benenson I., Torrens P.M., Geosimulation; Automata-based modeling of urban phenomena, Wiley, 2004.

[BRU 92] BRUnet R, FERRAS R., THERY H., Les mots de la géographie, Collection Dynamiques du territoire, RECLUS-La documentation Française, Paris, 1992.

[CHA 95] CHARre J., Statistique et territoire, Collection espaces mode d'emploi, RECLUS, Montpellier, 1995.

[CHE 95] Cheylan J-P., Lardon S., Mathian H., Sanders L., 1995, Les problématiques de l'espace et du temps et les SIG, Revue Internationale de Géomatique, vol.4, n³-4, p.287-305.

[FOT 91] Fotheringham A.S., Wong D.W.S., «The modifiable areal unit problem in multivariate statistical analysis », Environment and planning A, p. 1025-1044, 1991.

[HAG 65] HAGGETT; P., Locational analysis in modern geography, London; Arnold, 1965.

[LON 96] LONGLEY P., BATTY M., “Analysis, modelling, forecasting and GIS technology”, in Longley P., Batty M. (eds.), Spatial Analysis: Modelling in a GIS Environment, p.1-15, Wiley, 1996

[MAG 06] MAGUIRE D., BATTY M., GOODCHILD M., GIS, Spatial Analysis, and Modeling, ESRI Press, 2006

[POR 95] PORTUGALI J., « Towards cognitive approach to urban dynamics », Environment and Planning B: Planning and Design, volume 31(4), p. 589-613, 2004.

[PUM 97] PUMAin D., SAINT-JULIEN T., Analyse spatiale (1), collection CURSUS, Armand Colin, Paris, 1997.

[WHI 97] WHITE R., ENGELEN G., "Cellular automata as the basis of integrated dynamic regional modeling", Environment and Planning B, 24, p. 235-246, 1997. 